Numerical i nvesti gati on of the effect of cat hode hol der shape on haf ni um cat hode evaporation for an oxygen pl asma cutting ar c

\begin{tabular}{|l|l|}
\hline 著者 & $\begin{array}{l}\text { Nguyen Phi Long, Yasunor i Tanaka, Yoshi hi ko } \\
\text { Uesugi, Yoshi hi ro Yanaguchi }\end{array}$ \\
\hline $\begin{array}{l}\text { j our nal or } \\
\text { publ i cat i on t i t l e }\end{array}$ & Journal of Physi cs D: Appl i ed Physi cs \\
\hline vol une & 46 \\
\hline number & 22 \\
\hline page range & 224012 \\
\hline year & $2013-06-05$ \\
\hline URL & ht t p: //hdl . handl e. net /2297/35110 \\
\hline
\end{tabular}




\title{
Numerical investigation of the effect of cathode holder shape on hafnium cathode evaporation for oxygen plasma cutting arc
}

\author{
Nguyen Phi Long ${ }^{1,3}$, Yasunori Tanaka ${ }^{1}$, Yoshihiko Uesugi ${ }^{1}$, and \\ Yoshihiro Yamaguchi ${ }^{2}$ \\ ${ }^{1}$ Division of Electrical Engineering and Computer Science, Kanazawa University, \\ Kakuma, Kanazawa 920-1192, Japan \\ ${ }^{2}$ Komatsu Industries Corporation, Japan \\ E-mail: philong1980@gmail.com
}

\begin{abstract}
The effects of the cathode holder shape on a plasma cutting arc were investigated using a two dimensional thermo-fluid model developed for arc plasmas with the consideration of the hafnium cathode evaporation. The shape of the cathode holder shape was defined a convex structure which protrudes from the surface of the cathode holder. Results showed that the vortex of gas flow near the hafnium cathode was created by the effect of the convex cathode holder. The temperature of the cathode surface decreased markedly with the higher protrusion of the convex cathode holder by a rapid clockwise rotation of gas flow. The presence of gas flow vortex toward to the cathode surface reduces the mass fraction of hafnium vapor near the surface cathode. Finally, the total amount of mass loss of the hafnium evaporation was predicted to decrease markedly with the effect of the convex cathode holder.
\end{abstract}

Submitted to: J. Phys. D: Appl. Phys.

${ }^{3}$ Author to whom any correspondence should be addressed. 


\section{Introduction}

The plasma arc cutting (PAC) process is a technique widely used for cutting different metals with high-speed and high-accuracy in the industrial fields. An arc plasma is established between the electrode in the plasma torch and a work-piece. Cathode erosion in the plasma arc cutting is one issue that still introduces a limit on performance improvement when using a hafnium cathode and an oxygen gas plasma. It is necessary to understand in detail interactions between the electrode and the arc plasma as well as the arc cathode erosion on performance improvement and lifetime of plasma arc cutting devices.

A summary of several investigations related to plasma arc cutting processes can be found in topical reviews $[1,2]$. During operation of a plasma arc cutting torch, the molten material is ejected from the surface of cathode insert [3]. A significant amount of the material deposits on the inside surface of the nozzle and also changes the geometry of the cathode. Thus, the shape of the cathode insert surface changes rapidly until reaching the preferred concave shape at steady state [4]. As for the cathode with concave surface insert used in a plasma arc torch, it has been shown in patent literature $[4,5]$ that the curvature of this preferred concave shape is a function of the current level of the torch, and the diameter of the insert and the gas flow pattern in the torch. Use of this concave insert reduces deposition of the cathode material on the nozzle, and it thereby reduces nozzle wear in the torch. On the other hand, the results of report [6] suggested that the arc plasma temperature is decreased by heavy deposits of eroded cathode material on the nozzle after the large crater forms in the cathode, and the cut quality also decreases in case of cathode pit wears. Other literatures $[7,8]$ reported a start-up erosion mechanism by using high-speed camera imaging. A comparison of the arc behaviour during the start-up phases of new or used electrodes has been investigated with different electrode surface shapes. The new ones, both with or without the initially shaped emission surface, are characterized by a quite short transient phase towards stabilization of the arc column at the center of the Hf surface with a smooth transition event without the massive ejection of molted Hf-based particles. In contrast, the used electrodes are characterized by a quite long transient phase with the ejection of molten particles.

In addition, multiple reports of studies investigating the process parameters related to plasma arc cutting have been published [9]-[14]. Nemchinsky [9] confirmed that swirling the gas enhances the axial gas flow from the cathode. This enhanced axial gas flow sweeps the evaporated particles away from the cathode and thus increases the net evaporation rate. Another experimental result indicated the importance of the flow pattern close to the cathode $[1,10]$ : The erosion rate decreases with time for long arc duration tests, because there exists a stagnation zone inside the crater of the cathode insert. The probability of the evaporated atom/ion returning to the cathode is increased by the slowing down of the gas flow near the cathode and thus decreases the net erosion rate. On the other hand, our previous works $[13,14]$ have already used 
a numerical model for a fundamental study of the effect of various swirl gas angle and process parameters on behaviour of hafnium cathode evaporation in a plasma cutting arc torch. Results have shown that a larger swirl gas angle causes a high-temperature plasma area in front of the cathode surface shrunken in a radial direction. The flow patterns changed in the opposite direction in front of the cathode, and induced much hafnium vapor transportation along the center axis direction at a large swirl angle. This supports the experimental results of higher evaporation rate of the cathode.

As discussed above, the geometry of the electrode including cathode insert has a marked effect on the characteristics of arc plasma. It is, however in general, too much complicated to investigate the interaction between thermal plasma and surface cathode insert shape such as evaporation of the cathode material. Experimental investigations of a plasma arc cutting torches with different shapes of the hafnium cathode insert are very difficult because of the high running cost of plasma arc cutting. Also, we confirmed that the $50 \%$ running cost of the device is due to the eroded hafnium cathode exchange. Another approach for the geometry of the electrode is to investigate the shape of a cathode holder, not a cathode insert. This holder structure may affect the characteristics of arc plasma, especially gas flow pattern. Thus, this can also control the amount of cathode material evaporation. For this purpose, numerical modeling is extremely important to predict the erosion amount of hafnium cathode in different holder geometry and to enhance the performance and the lifetime of plasma arc cutting devices.

The aim of this work is to investigate the effects of cathode holder shape, not the cathode insert itself, on hafnium cathode evaporation by a developed two-dimensional numerical model for oxygen arc plasma in a torch. Arc cathode erosion is a very important but still not completely understood phenomenon in plasma arc cutting processes. The cathode erosion occurs not only during arc cutting but also during the processes of switching the arc on and arc off $[2,3]$. This paper paid attention to steady state evaporation of cathode material during arc cutting because it is also important for long operation of the plasma arc cutting torch. The shape of the cathode holder shape was defined a convex structure which protrudes from the surface of the cathode holder. The governing equations for mass, momentum, and energy transfer from the evaporated material in the arc plasma were used to obtain the simulation results which have been set up in the previous works $[13,14]$. This developmental model incorporates evaporation phenomena of the hafnium cathode and the re-deposition of hafnium vapour on the cathode surface [9]. Using this model, the temperature field, gas flow pattern, and mass fraction of hafnium vapour inside of plasma cutting torch as well as the total amount of mass loss of the hafnium cathode caused by evaporation were obtained with different the shapes of the cathode holder. 


\section{Mathematical model}

\subsection{The shape of the cathode holder}

Figure 1 portrays a schematic diagram of the cross section of the DC plasma arc cutting (PAC) torch used in this work. The PAC torch has axisymmetric structure. The copper electrode holder has a hafnium tip insert of $1.27 \mathrm{~mm}$ in diameter, at the center of the holder. Oxygen, which is the plasma gas used in the experiments, is supplied from the inlet by swirling gas flow. The distance between the cathode surface and nozzle bore exit is approximately $8 \mathrm{~mm}$. The arc plasma is constricted by the copper nozzle. The nozzle outlet has $1.33 \mathrm{~mm}$ in diameter.

It is considered that the shape of the cathode holder can affect the gas flow pattern and the arc plasma temperature profiles, which also influences the Hf cathode erosion rates. In this paper, thus, the calculation is performed for different the shapes of the cathode holder shape, which are shown in figure 2. In this figure, holder $\mathrm{O}$ is the original flat surface holder, which is usually used in the experiments. The holders A and B indicate two of "convex cathode holder", which has a protruded shape as seen in figure 2. The shape of these holders is defined by a distance $\ell$ from the flat surface of the cathode and the top of the convex cathode holder at the radial position of $1.6 \mathrm{~mm}$. The holder A has the distance $\ell$ of $0.4 \mathrm{~mm}$, while the holder $\mathrm{B}$ has the distance $\ell$ of $0.5 \mathrm{~mm}$. The convex shape of the holders A and B are formed as a part of an arc of an ellipse.

\subsection{Assumptions}

The developed two-dimensional numerical model assumes the followings. The plasma is in a steady state and axisymmetric. As the maximum value of the Reynolds number found in the nozzle was estimated to be around 1800, we assume the gas flow to be laminar in the nozzle. For simplicity in modeling, the thermal plasma is under local thermal equilibrium (LTE) conditions, which means that the electron and heavy-particle temperatures are equal under chemically equilibrium condition. The plasma is optically thin the wavelengths greater than $200 \mathrm{~nm}$. For the wavelengths of less than $200 \mathrm{~nm}$, $20 \%$ of the total emission coefficient is accounted for radiation loss to consider the effective light absorption [16, 17]. The effect of swirl gas flow is considered. A simple energy balance equation is set up on the cathode surface to determine the hafnium evaporation process and to calculate the mass loss of the hafnium evaporation. The evaporation amount of the hafnium cathode was predicted considering the evaporation flux estimated using the saturation vapour pressure and the re-deposition flux. The arc radiation was estimated according to the emission coefficient as described later.

The electric field has only an axial component, whereas the magnetic field has only an azimuthal component for simplicity. In plasma cutting, the arc is much longer, and the cathode attachment region tends to be larger. Therefore, the radial component of current density is expected to be much smaller. This is similar to the case of the 
high voltage circuit breaker [15]. In tungsten inert gas welding, the radial component of current density is relatively large, since the arc radius changes rapidly from well under $1 \mathrm{~mm}$ at cathode tip to 3-5 $\mathrm{mm}$ at the anode and the arc length usually only 3-5 $m m$. Therefore it is important to include the axial Lorentz force component. Further, the focus of the paper is on the effects of the cathode holder geometry on the gas flow pattern around the cathode hafnium evaporation process. The shape of cathode itself is not changed. There will be some changes to the current density due to changes in the temperature at the edge of the arc column, but it would not expect these to be critical.

\subsection{Governing equations for gas and plasma}

On the basis of the assumptions described above, the gas and arc plasma are governed by the following equations:

- Mass conservation:

$$
\nabla \cdot(\rho \boldsymbol{u})=S_{\mathrm{p}}^{\mathrm{Hf}}
$$

- Momentum conservation:

Axial:

$$
\begin{aligned}
& \nabla \cdot(\rho \boldsymbol{u} u)=-\frac{\partial p}{\partial z}+\nabla \cdot(\eta \nabla u)+\nabla \cdot\left(\eta \frac{\partial \boldsymbol{u}}{\partial z}\right) \\
& -\frac{2}{3} \frac{\partial}{\partial z}(\eta \nabla \cdot \boldsymbol{u})
\end{aligned}
$$

Radial:

$$
\begin{aligned}
& \nabla \cdot(\rho \boldsymbol{u} v)=-\frac{\partial p}{\partial r}+\nabla \cdot(\eta \nabla v)+\nabla \cdot\left(\eta \frac{\partial \boldsymbol{u}}{\partial r}\right) \\
& -2 \eta \frac{v}{r^{2}}+\frac{\rho w^{2}}{r}-\frac{2}{3} \frac{\partial}{\partial r}(\eta \nabla \cdot \boldsymbol{u})-\mu_{0} \sigma E_{\mathrm{z}} H_{\theta}
\end{aligned}
$$

Swirl:

$$
\nabla \cdot(\rho \boldsymbol{u} w)=\nabla \cdot(\eta \nabla w)-\frac{\rho v w}{r}-\frac{\rho w}{r^{2}} \frac{\partial}{\partial r}(r \eta)
$$

- Energy conservation:

$$
\begin{aligned}
& \nabla \cdot(\rho \boldsymbol{u} h)=\nabla \cdot\left(\frac{\lambda}{C_{\mathrm{P}}} \nabla h\right)+\boldsymbol{u} \cdot \nabla p+\sigma\left|E_{\mathrm{z}}\right|^{2} \\
& -P_{\mathrm{rad}}-L_{\mathrm{v}} S_{\mathrm{p}}^{\mathrm{Hf}}
\end{aligned}
$$

- Mass conservation for Hf vapour:

$$
\nabla \cdot\left(\rho \boldsymbol{u} Y_{\mathrm{Hf}}\right)=\nabla \cdot\left(\rho D_{\mathrm{Hf}-\mathrm{O}_{2}} \nabla Y_{\mathrm{Hf}}\right)+S_{\mathrm{p}}^{\mathrm{Hf}}
$$

- Ohm's law:

$$
E_{\mathrm{z}}=\frac{I}{\int 2 \pi r \sigma d r}
$$

- Ampere's law:

$$
H_{\theta}=\frac{1}{r} \int_{0}^{r} \sigma E_{\mathrm{z}} \xi d \xi
$$


In those equations, $\boldsymbol{u}$ is the gas flow vector; $r$ and $z$ respectively denote the radial and axial positions. Additionally, $u, v$, and $w$ respectively represent the axial, radial, and swirl gas flow velocities. The following are other important variables: $\rho$, mass density; $h$, enthalpy; $p$, pressure; $\eta$, viscosity; $\sigma$, electrical conductivity; $\lambda$, thermal conductivity; $C_{\mathrm{P}}$, specific heat at constant pressure; $P_{\mathrm{rad}}$, radiation loss; $\xi$, radial coordinate; $E_{\mathrm{z}}$, electric field in axial direction; $H_{\theta}$, magnetic field in azimuthal direction ; $Y_{\mathrm{Hf}}$, mass fraction of $\mathrm{Hf}$ vapour; $D_{\mathrm{Hf}-\mathrm{O}_{2}}$, effective diffusion coefficient of $\mathrm{Hf}$ vapour in $\mathrm{O}_{2} ; L_{\mathrm{v}}$, latent heat for evaporation; $S_{\mathrm{p}}^{\mathrm{Hf}}$, the mass production rate because of evaporation; and $I$, the total electric current. In equation (5), we neglected gradient term of electron enthalpy for simplicity.

\subsection{Governing equations for electrodes}

Inside the solid and liquid, the energy conservation equation is established as

$$
0=\frac{\partial}{\partial z}\left(\frac{\lambda_{\mathrm{s}}}{C_{\mathrm{ps}}} \frac{\partial h_{\mathrm{s}}}{\partial z}\right)+\frac{1}{r} \frac{\partial}{\partial r}\left(r \frac{\lambda_{\mathrm{s}}}{C_{\mathrm{ps}}} \frac{\partial h_{\mathrm{s}}}{\partial z}\right)+\sigma_{\mathrm{s}}\left|E_{\mathrm{z}}\right|^{2}
$$

where $h_{\mathrm{s}}$ signifies the enthalpy of the solid/liquid, $C_{\mathrm{ps}}$ denotes the specific heat of the solid/liquid, $\lambda_{\mathrm{s}}$ stands for the thermal conductivity of the solid/liquid, and $\sigma_{\mathrm{s}}$ represents the electrical conductivity of the solid/liquid. The enthalpy of the solid is related to specific heat as shown below.

$$
\begin{aligned}
& h_{\mathrm{s}}\left(T_{\mathrm{s}}\right)=\int_{T_{0}}^{T_{\mathrm{s}}} C_{\mathrm{ps}}^{\mathrm{eff}} d T_{\mathrm{s}} \\
& C_{\mathrm{ps}}^{\mathrm{eff}}= \begin{cases}\frac{L_{\mathrm{m}}}{\Delta T}+C_{\mathrm{ps}} & \left(T_{\text {melt }}-\Delta T \leq T_{\mathrm{s}} \leq T_{\text {melt }}\right) \\
C_{\mathrm{ps}} & \text { (otherwise) }\end{cases}
\end{aligned}
$$

Therein, the following variables are used: $T_{\mathrm{s}}$, temperature of the solid/liquid; $T_{0}$, reference temperature $\left(T_{0}=300 \mathrm{~K}\right) ; T_{\text {melt }}$, melting temperature; $C_{\mathrm{ps}}^{\mathrm{eff}}$, effective specific heat; $L_{\mathrm{m}}$, latent heat for melting of solid; and $\Delta T$, step temperature for melting $(\Delta T=5$ $\mathrm{K})$.

In this model, interaction between the thermal plasma and solid is solved. Heat transfer to the surface cathode in this model can occur through the thermal energy of ions, the heating by surface recombination of ions, the energy loss by electron emission, and the thermal conduction from the arc plasma and the thermal radiation from the cathode. The heating of the cathode by radiation from the arc was neglected in comparison with the other energy fluxes for a simplified model. These are described by the following additional heat flux to the energy conservation equation for the cathode surface:

$$
\begin{aligned}
& q_{\mathrm{K}}=\frac{j_{\text {ion }}}{e}\left(\frac{3}{2} k T_{\mathrm{p}}+\epsilon_{\mathrm{ion}}-W_{\ell}\right) \\
& -\frac{j_{\mathrm{e}}}{e} W_{\ell}-\frac{\lambda_{\mathrm{pc}}}{\Delta z}\left(T_{\mathrm{c}}-T_{\mathrm{p}}\right)-\delta_{\ell} \sigma_{\mathrm{sb}}\left(T_{\mathrm{c}}^{4}-T_{\mathrm{a}}^{4}\right) .
\end{aligned}
$$

The current density, because of the thermionic emission, depends on the cathode temperature using the well-known Richardson-Dushman equation with the Schottky 
effect, which is given as presented below.

$$
\begin{aligned}
& j_{\mathrm{e}}=\alpha_{\ell} A T_{\mathrm{c}}^{2} \exp \left(-\frac{W_{\ell}}{k T_{\mathrm{c}}}\right) \exp \left(-\frac{e}{2 k T_{\mathrm{c}}} \sqrt{\frac{e E_{0}}{\pi \varepsilon_{0}}}\right) \\
& j_{\text {ion }}=\max \left(0, \sigma E_{0}-j_{\mathrm{e}}\right)
\end{aligned}
$$

In those equations, the following variables are used: $q_{\mathrm{K}}$, heat flux between the surface cathode and plasma; $T_{\mathrm{p}}$, temperature of the plasma contacting with the cathode; $T_{\mathrm{c}}$, temperature of the surface cathode contacting with the plasma; $T_{\mathrm{a}}$, ambient temperature, here $300 \mathrm{~K}$ as the wall temperature of nozzle; e, elementary charge; $k$, Boltzmann's constant; $\sigma_{\mathrm{sb}}$, Stefan-Boltzmann's constant; $A$, thermionic emission constant; $\varepsilon_{0}$, vacuum permittivity; $W_{\ell}$, work function of $\mathrm{Hf}$ and $\mathrm{Cu} ; \epsilon_{\text {ion }}$, ionization energy of oxygen ion; $\delta_{\ell}$, solid emissivity; (assumed to be 0.3 ); $\alpha_{\ell}$, material factor for thermionic emission; $j_{\mathrm{e}}$, thermionic emission current density of electron; $j_{\text {ion }}$, ion current density; $E_{0}$, electric field at the cathode surface; $\lambda_{\mathrm{pc}}$, thermal conductivity of cathode; and $\Delta z$, distance between the surface cathode and the center of the control volume. The present calculation neglected the radial components of current density and electric field for simplicity [15].

\subsection{Governing equations for evaporation flux}

The mass production rate attributable to evaporation $S_{\mathrm{p}}^{\mathrm{Hf}}$ is calculable approximately as

$$
S_{\mathrm{p}}^{\mathrm{Hf}}= \begin{cases}m_{\mathrm{Hf}}\left(\Gamma_{\mathrm{evp}}-\Gamma_{\mathrm{dep}}\right) \frac{\Delta S}{\Delta V} & \text { (neighbor to wall) } \\ 0 & \text { (otherwise) }\end{cases}
$$

Therein, $m_{\mathrm{Hf}}$ is the effective mass of Hf vapour, $\Gamma_{\text {evp }}$ is the mass flux of evaporated vapour, $\Gamma_{\text {dep }}$ is the mass flux of re-deposition, $\Delta S$ is the surface of evaporated Hf, and $\Delta V$ is the volume of the control volume.

The mass flux of the evaporated vapour $\Gamma_{\text {evp }}$ was calculated using the following Hertz-Knudsen relation, as

$$
m_{\mathrm{Hf}} \Gamma_{\mathrm{evp}}=m_{\mathrm{Hf}} \frac{1}{4} \frac{P_{\mathrm{v}}}{k T_{\mathrm{c}}} \sqrt{\frac{8 k T_{\mathrm{c}}}{\pi m_{\mathrm{Hf}}}}=P_{\mathrm{v}} \sqrt{\frac{m_{\mathrm{Hf}}}{2 \pi k T_{\mathrm{c}}}},
$$

where $P_{\mathrm{v}}$ is the saturation vapour pressure of hafnium vapour.

The saturation vapour pressure $P_{\mathrm{v}}$ was evaluated using the Clausius-Clapeyron relation as

$$
P_{\mathrm{v}}= \begin{cases}P_{1 \text { atm }} \exp \left[\frac{L_{\mathrm{v}}}{R_{\mathrm{Hf}}}\left(\frac{1}{T_{\text {boil }}}-\frac{1}{T_{\mathrm{c}}}\right)\right] & \left(T_{\mathrm{c}} \geq T_{\text {melt }}\right) \\ P_{\text {melt }} \frac{T_{\mathrm{c}}-T_{\text {melt }}+\Delta T}{\Delta T} & \left(T_{\mathrm{c}}<T_{\text {melt }}\right)\end{cases}
$$

Therein, $R_{\mathrm{Hf}}$ denotes the gas constant of hafnium vapour, $P_{1 \mathrm{~atm}}$ is the standard pressure, and $T_{\text {melt }}$ is the melting temperature of Hf with $\Delta T=0.1 \mathrm{~K}$. 
The mass flux of re-deposition vapour $\Gamma_{\text {dep }}$ was calculated as

$$
m_{\mathrm{Hf}} \Gamma_{\mathrm{dep}}=m_{\mathrm{Hf}} \frac{1}{4} \frac{\rho Y_{\mathrm{Hf}}}{m_{\mathrm{Hf}}} \sqrt{\frac{8 k T_{\mathrm{c}}}{\pi m_{\mathrm{Hf}}}}=\rho Y_{\mathrm{Hf}} \sqrt{\frac{k T_{\mathrm{c}}}{2 \pi m_{\mathrm{Hf}}}} .
$$

\subsection{Thermodynamic and transport properties of the solid material, transport properties of oxygen plasma and Hf vapour}

Table 1 shows the thermodynamic properties of the solid materials used in this work. The characteristics of Hf with the low specific heat, low thermal conductivity, high melting and boiling temperature, and with other features, are compared with the characteristics of $\mathrm{Cu}$.

Thermodynamic and transport properties of oxygen thermal plasma with hafnium vapour were calculated under the local thermodynamic equilibrium assumption as follows. First, the equilibrium compositions of oxygen and hafnium vapour at different pressures were calculated as a function of the temperature from 300 to $30000 \mathrm{~K}$ by minimization of Gibbs' free energy of the system. The species included in the calculations were as follows: for oxygen, $\mathrm{O}_{3}, \mathrm{O}_{2}, \mathrm{O}_{2}^{-}, \mathrm{O}_{2}^{+}, \mathrm{O}, \mathrm{O}^{+}, \mathrm{O}^{2+}$, and e; for hafnium, $\mathrm{Hf}, \mathrm{Hf}^{+}$, and e. Using the calculated equilibrium compositions, we computed the thermodynamic properties like enthalpy $h$, specific heat $C_{\mathrm{P}}$, and mass density $\rho$. Transport properties such as the electrical conductivity $\sigma$, the thermal conductivity $\lambda$, and the viscosity $\eta$ were calculated based on the first-order approximation of the Chapman-Enskog method using the calculated equilibrium compositions and collision integrals [18]-[23]. The collision integrals used in the calculation are classified into a momentum transfer cross section and viscosity cross sections for interactions between oxygen species and hafnium species. The required collision integrals for interactions between oxygen species were calculated using the method given by [20,23]. The collision integrals for Hf-Hf interactions were obtained by using exponential repulsive potential given by Abrahamson [24] and Monchick' table [25]. The collision integrals for Hf$\mathrm{Hf}^{+}$interactions were calculated considering the effects of resonant charge exchange [23], which is interpolated from the Rapp's data versus ionization potential [26]. For interactions of e-Hf, the collision integrals estimated by the hard-sphere method. In addition, the thermodynamic and transport properties of the mixture of hafnium vapour and oxygen were found by using a mixture law based on the concentration ratio. The resulting properties of the gas mixture were applied to thermo-fluid analysis as functions of temperature.

The effective diffusion coefficient of $\mathrm{Hf}$ vapour in oxygen $D_{\mathrm{Hf}-\mathrm{O}_{2}}$ was calculated from the following effective binary diffusion coefficient approximation for simplicity [19, 22]:

$$
D_{\mathrm{Hf}-\mathrm{O}_{2}}=\frac{3}{8} \frac{k T}{p}\left[\frac{\pi k T\left(m_{\mathrm{O}_{2}}+m_{\mathrm{Hf}}\right)}{2 m_{\mathrm{O}_{2}} m_{\mathrm{Hf}}}\right]^{\frac{1}{2}} \frac{1}{Q_{\mathrm{Hf}-\mathrm{O}_{2}}}
$$

where $k$ is the Boltzmann constant, $m_{\mathrm{O}_{2}}$ is the mass of $\mathrm{O}_{2}$ atom, $m_{\mathrm{Hf}}$ is the effective mass of $\mathrm{Hf}$ vapour depending on particle composition of $\mathrm{Hf}$ vapour, $Q_{\mathrm{Hf}-\mathrm{O}_{2}}$ is the effective 
momentum transfer cross section between $\mathrm{O}_{2}$ and $\mathrm{Hf}$ vapour, which was estimated from the hard-sphere method.

The radiation loss $P_{\text {rad }}$ was computed while taking account of atomic and ionic spectral lines and the continuous spectrum including the bremsstrahlung and the recombination radiation by using the following classical expressions [23]:

$$
\begin{aligned}
P_{\mathrm{rad}} & =\frac{4 \pi}{\left(4 \pi \varepsilon_{0}\right)^{3}} \frac{16 \pi e^{6} n_{\mathrm{e}}}{3 c^{3}\left(6 \pi m_{\mathrm{e}}^{3} k T_{\mathrm{e}}\right)^{\frac{1}{2}}} \frac{4 k T_{\mathrm{e}}}{h_{\mathrm{p}}} \\
& +\sum_{\text {all-lines }} \frac{n_{\ell}}{4 \pi Z_{\ell}} \frac{h_{\mathrm{p}} c}{\lambda_{\ell}} g_{\ell} A_{\ell} \exp \left(-\frac{E_{\ell}}{k T_{\mathrm{e}}}\right)
\end{aligned}
$$

where $c$ is the velocity of light, $Z_{\ell}$ is the partition function of speccies $\ell, n_{\ell}$ the number density of speccies $\ell, h_{\mathrm{p}}$ the Planck's constant, $g_{\ell}$ the statistical weigh, $A_{\ell}$ the transition probability, $E_{\ell}$ the energy of the upper level, $\lambda_{\ell}$ the wavelength. All data sources used in the calculation were taken from $[27,28,29]$.

\subsection{Boundary conditions}

Figure 1 shows the computational domain where O-A-B-C-D-E-F-G-H-I-K-L define the calculation space inside which fluid parameters and scalar quantities were evaluated. The two-dimensional space coordinates are $\mathrm{O}(0,0), \mathrm{A}(0,9.3), \mathrm{B}(9.3,0.67), \mathrm{C}(9.3$, 4.3), D (0, 4.3), E (0, 3.4), F (1.4, 2.7), G (1.4, 0.63), H (1.4, 0), I (3.8, 1.3), K (5.1, $1.3)$ and $\mathrm{L}(6.3,0.67)$ where the unit is millimeters. The non-uniform grid system with a size of 98 nodes in the vertical direction and 185 nodes in the horizontal direction is applied to the analytic space. The non-slip condition was considered on all the boundary walls between solid and gas HG, GF, EF, DI, IK, IL and LB. On axis OA, the axial symmetry condition is applied. The following conditions were set at the boundary of the computational domain:

At the centre axis (OA):

$$
\frac{\partial u}{\partial r}=0 ; \quad v=0 ; \quad \frac{\partial w}{\partial r}=0 ; \quad \frac{\partial T}{\partial r}=0 .
$$

At the copper wall (OE, DCB):

$$
u=0 ; \quad v=0 ; \quad w=0 ; \quad T=300 \mathrm{~K} .
$$

At the outlet $(\mathrm{AB})$ :

$$
\frac{\partial(\rho u)}{\partial z}=0 ; \quad \frac{\partial(\rho v)}{\partial z}=0 ; \quad \frac{\partial(\rho w)}{\partial z}=0 ; \quad \frac{\partial T}{\partial z}=0 .
$$

At the inlet (ED):

$$
u=u_{\text {in }} \quad v=0 ; \quad w=w_{\text {in }} ; \quad T=300 K ; \quad p=p_{\text {in }} .
$$

Oxygen gas is injected from the inlet ED with a swirl component that can be expressed by the swirl gas angle [13].

In this paper, the calculation model is performed for different the shapes of the cathode holder, as shown in figure 2. In addition, the operating parameters for 
simulation are that the swirl gas angle is fixed at 15 deg. The arc current of $100 \mathrm{~A}$ and gas flow rate of $20 \mathrm{slm}$ are fixed in this calculation model. Pressure at the inlet is also fixed at 0.9 MPa. For simplicity, the boundary shape is assumed not to change by melting and evaporation. The SIMPLE method described by Patankar [30] was used for the calculation scheme to solve the governing equations described in the previous section.

\section{Calculation Results}

\subsection{Effects of the convex cathode holder on the arc plasma temperature}

Figure 3 portrays the temperature distributions of the arc plasma with the shapes of the cathode holder of $\mathrm{O}, \mathrm{A}$, and $\mathrm{B}$. The isothermal color fringe for temperature is indicated in logarithm scale. The arc temperature profiles at axial position $z>3 \mathrm{~mm}$ are almost the same to the three shapes of the cathode holder. On the other hand, it is apparent that the arc root near the cathode has expanded in the radial direction with the effect of the convex cathode holder. The arc root behaviour tends to move to the surface of the cathode holder with the higher protrusion of the convex cathode holders A and B. Another noticeable point is that the temperature inside the copper cathode holder is decreased in cases of the convex holders compared to the holder O.

Figure 4 shows the radial distribution of the arc plasma temperature just near the cathode surface. The arc temperature profiles have a local minimum at the center axis, which is due to the effect of swirl gas flow as described in our previous work [13]. The important point in figure 4 is that in cases of the holder $\mathrm{A}$ and $\mathrm{B}$, the arc temperature at the radial position $r<0.4 \mathrm{~mm}$ is lower compared with that for holder $\mathrm{O}$. In addition, the temperature for holder A and B has wider profiles than holder O. Figure 5 depicts the radial distribution of the current density in the arc plasma near the cathode surface. The current density also has similar profiles of the temperature shown in figure 4, against the shape of the cathode holder. These results suggest that the decreased arc temperature and current density with the effect of the convex cathode holder occur at the same radial position around $r<0.4 \mathrm{~mm}$. An increase in the protrusion of the convex cathode holder induces the expansion of the arc root resulting in a low current density in the immediate vicinity of the cathode, which decreases the efficient heating of the cathode surface and therefore a descending in the hafnium insert temperature.

The cathode surface temperature determines the thermionic electron emission current and Hf evaporation flux, which is crucial for erosion process of the cathode. The radial distribution of the surface temperature of the hafnium cathode for different the shapes of the cathode holder is presented in figure 6 . The hafnium cathode surface temperature is decreased markedly around radial position $r<0.4 \mathrm{~mm}$ with the effect of the convex cathode holder. The higher protrusion of the convex cathode holder results in the lower hafnium surface temperature. This decrease in the surface temperature is related mainly to arc behaviour by the changing of the convex cathode holder, as 
described before. This arc behaviour is expected to decrease the heat transferred to the surface of the hafnium cathode by the effect plasma gas flow. Furthermore, there is no significant change in the holder surface temperature, which remains lower than the melting temperature of copper even though the effect of the convex cathode holder.

\subsection{Gas flow pattern affected by the convex cathode holder}

The shape of the cathode holder affects the gas flow pattern, which markedly influences the arc behaviour including the arc temperature profiles, and then the Hf cathode surface temperature. Figure 7 depicts a comparison of gas flow fields obtained with the shapes of the cathode holder of $\mathrm{O}, \mathrm{A}$, and $\mathrm{B}$. As a result described in the flat surface holder $\mathrm{O}$, the gas flow in front of the Hf cathode surface moves away from the cathode by the effect of swirling gas flow. This gas flow from the cathode is reduced markedly using the convex cathode holder A. In the cathode holder B case, it is noteworthy that a circular vortex appears in front of the Hf cathode in which the flow direction is mainly toward the cathode and moves in the direction against that of the swirling gas flow. Figure 8 also shows the axial distribution of velocity along the center axis. It is indicated that the velocity has a negative value in the axial position close to the cathode in the case of holder B. This negative axial velocity shows a considerable rise with an increase in the protrusion of the convex cathode holder.

Furthermore, the $\theta$-component of the vorticity $\omega_{\theta}$ is simply calculated as following equation:

$$
\omega_{\theta}=[\nabla \times \boldsymbol{u}]_{\theta}=\frac{\partial u}{\partial z}-\frac{\partial v}{\partial r}
$$

where $\theta$-component means the component perpendicular to a $r-z$ two dimensional plane. The quantity $\omega_{\theta}$ indicates the local spinning motion of a fluid at each position. In this paper, negative $\omega_{\theta}$ shows the clockwise spinning motion of a fluid, while positive $\omega_{\theta}$ is the counter-clockwise spinning motion. Figure 9 presents the spatial distribution of $\omega_{\theta}$ with different the shapes of the cathode holder. In case of the flat surface holder $\mathrm{O}$, the high counter-clockwise rotation near the center axis occurs in the nozzle throat region around the axial position of $5 \mathrm{~mm}<z<7 \mathrm{~mm}$, resulting in the high constricted arc plasma and quality of the speed cutting. Near the hafnium cathode, the value of the vorticity is low for holder O. On the other hand, it appears a clockwise rotation in the vicinity of the hafnium cathode with convex cathode holders $\mathrm{A}$ and $\mathrm{B}$. The acceleration of the clockwise rotation of gas velocity is more evident in the case of cathode holder B.

Calculations showed that the convex holder cathodes not only reduces the gas flow velocity from the cathode surface, but also makes a vortex in the vicinity of the cathode surface moving in the opposite direction. For this reason, the arc root moves more into the holder cathode region. On the other hand, the rapid clockwise rotation velocity against the effect of swirling gas flow from the cathode is expected to provide more convective heat transfer from the hafnium cathode surface, resulting in the decreasing of cathode surface temperature. The presence of this vortex can be related to the evaporation amount of the hafnium cathode which is discussed in the next section. 


\subsection{Effects of the convex cathode holder on evaporation amount of hafnium cathode}

Figure 10(a) and (b) show the distributions of the mass fraction of hafnium vapour with or without the consideration of redeposition for different the shapes of the cathode holder. Results demonstrated that the mass fraction of hafnium vapour with the convex cathode holder decreases markedly in comparison with the flat holder O. This is because the current density, and therefore the cathode surface temperature, is lower for holder A and $\mathrm{B}$ than for holder $\mathrm{O}$. The consideration of redeposition of hafnium vapour reduces the mass fraction of hafnium vapour in three cases of the shape of the cathode holder.

The results from the shapes of the cathode holder of $\mathrm{O}$ and $\mathrm{B}$ show that the high hafnium vapour is concentrated in the radial position with high current density near the cathode surface, and it is transported to the downstream region of the plasma torch along the central axis by convection. In case of holder B, as significant effect on the convex cathode holder, the hafnium vapour spreads out a larger area near cathode surface, according to gas flow vortex. Moreover, the hafnium vapour without the consideration of redeposition covers the entire surface of the hafnium cathode by higher concentration of hafnium vapour.

As described in this paper, the net evaporation mass flux $m_{\mathrm{Hf}} \Gamma_{\text {net }}\left[\mathrm{kg} /\left(\mathrm{m}^{2} \mathrm{~s}\right)\right]$ can be estimated as

$$
m_{\mathrm{Hf}} \Gamma_{\mathrm{net}}=m_{\mathrm{Hf}}\left(\Gamma_{\mathrm{evp}}-\Gamma_{\mathrm{dep}}\right) .
$$

The mass flux of hafnium vapour ejected from the cathode for different the shapes of the cathode holder are given along the radial direction in figure 11. Comparison of net evaporation mass flux between the convex cathode holders A, B and the flat cathode holder $\mathrm{O}$ indicated that the net mass flux effect of the convex holder has a decrease markedly in the entire surface of the hafnium cathode. This is mainly due to the lower temperature of the cathode surface as described in figure 4. The appearance of the negative net evaporation flux around the arc fringe in vicinity of the hafnium cathode is dominated by the evaporated atoms returning to cathode surface.

In addition, the total mass loss attributable to evaporation of the hafnium cathode was estimated from these results obtained using the following equation:

$$
M_{\mathrm{Hf}}=\int_{0}^{\infty} m_{\mathrm{Hf}}\left(\Gamma_{\mathrm{evp}}-\Gamma_{\mathrm{dep}}\right) 2 \pi r d r .
$$

Figure 12 shows the calculated total amount of mass loss of the hafnium cathode different the shapes of the cathode holder comparing with no redeposition of hafnium vapour. Showing the same trend as that of the mass flux of hafnium vapour, the effect of the convex holder cathode reduces the amount of mass loss of the hafnium cathode evaporation. The higher protrusion of the convex cathode holder induces the lower amount of mass loss, although this progress decreases slowly in comparison of the convex cathode holder $\mathrm{A}$ and $\mathrm{B}$. The reason for a decrease in the amount of mass loss is that the gas flow vortex close to the cathode surface is present to move in the clockwise direction. This gas flow vortex motion toward to the hafnium cathode surface reduces the probability of evaporated hafnium escaping from the cathode surface, and 
also facilitates the evaporated atoms returning to cathode surface. In addition, as shown in this figure, the redeposition of hafnium vapour decreases the mass loss of the hafnium cathode.

\section{Conclusions}

Numerical simulations were performed to study the influence of the shape of the cathode holder on hafnium cathode evaporation for oxygen plasma cutting arc torch in this paper. We have developed a two-dimensional thermo-fluid model for plasma cutting arcs with different convex cathode holders. The simulated results show that the convex holder cathode not only reduces the gas flow velocity from the cathode surface, but also makes a vortex in the vicinity of the cathode surface moving in the opposite direction. The cathode surface temperature decreases markedly with the higher protrusion of the convex cathode holder, resulting in a rapid clockwise rotation of gas flow. In addition, the mass flux and total amount of mass loss of the hafnium cathode were predicted to decrease significantly with the effect of the convex cathode holder. This may result from the presence of gas flow vortex toward to cathode surface, which reduces the probability of evaporated hafnium escaping from the cathode surface and also facilitates the evaporated atoms returning to cathode surface.

\section{References}

[1] Nemchinsky V A, and Severance W S 2006 What we know and what we do not know about plasma arc cutting J. Phys. D: Appl. Phys. 39 R423-8.

[2] Colombo V, Concetti A, Ghedini E, Dallavalle S, and Vancini M 2009 High-speed imaging in plasma arc cutting: a review and new developments Plasma Sources Sci. Technol. 18023001.

[3] Peters J, Yin F, Borges C F M, Heberlein J, and Hackett C 2005 Erosion mechanisms of hafnium cathodes at high current J. Phys. D: Appl. Phys. 38 1781-94.

[4] Luo L et al 1997 Electrode for a plasma arc torch Hypertherm Inc. Patent US 5,601,734.

[5] Sakuragi S 1993 Cathode structure of plasma arc torch Kabushiki Kaisha Komatsu. Patent US $5,177,338$.

[6] Peters J, Bartlett B, Lindsay J, and Heberlein J 2008 Relating spectroscopic measurements in a plasma cutting torch to cutting performance Plasma Chem Plasma Process 28 331-352.

[7] Colombo V, Concetti A, Ghedini E, and Nemchinsky V 2010 High-speed imaging investigation of transition phenomena in the pilot arc phase in Hf cathodes for plasma arc cutting Plasma Sources Sci. Technol. 19065025.

[8] Colombo V, Concetti A, Ghedini E, Rotundo F, Sanibondi P, Boselli M, Dallavalle S, Gherardi M, Nemchinsky V, and Vancini M 2010 Adavances in plasma arc cutting technology: The experimental part of an integrated approach Plasma Chem. Plasma Process. 3241126.

[9] Nemchinsky V A, and Showalter M S 2003 Cathode erosion in high-current high-pressure arc $J$. Phys. D: Appl. Phys. 36 704-12.

[10] Nemchinsky V A 2002 Cathode erosion rate in high-pressure arcs. Influence of swirling gas flow IEEE Trans. Plasma Sci. 30 2113-6.

[11] Freton P, Gonzalez J J, Gleizes A, Camy Peyret F, Caillibotte G, and Delzenne M 2002 Numerical and experimental study of a plasma cutting torch J. Phys. D: Appl. Phys. 35 115-31.

[12] Zhou Q, Yin H, Lin H, Xu X, Liu F, Guo S, Chang X, Guo W, and Xu P 2009 The effect of 
plasma-gas swirl flow on a highly constricted plasma cutting arc J. Phys. D: Appl. Phys. 42 095208.

[13] Long N P, Tanaka Y, and Uesugi Y 2012 Numerical investigation of the swirl gas angle and arc current dependence on evaporation of hafnium cathode in a plasma cutting arc IEEE Trans. Plasma Sci. 40 no. 2, 497-504.

[14] Long N P, Katada Y, Tanaka Y, Uesugi Y and Yamaguchi Y 2012 Cathode diameter and operating parameter effects on hafnium cathode evaporation for oxygen plasma cutting arc J. Phys. D: Appl. Phys. 45435203.

[15] Wang W, Yan J D, Rong M, Murphy A B and Spencer J W 2013 Theoretical investigation of the decay of an SF6 gas-blast arc using a two-temperature hydrodynamic model J. Phys. D: Appl. Phys. 46065203.

[16] Boulos M I, Fauchais P, and Pfender E 1994 Thermal plasmas fundamentals and applications. I. Plenum Press.

[17] Tanaka Y Numada T, Kaneko S and Okabe S 2005 Thermodynamic and transport properties of polymer ablated vapors and influence of their inclusions on Ar induction thermal plasma temperature JSME International Journal Ser. B 48 417-24.

[18] Tanaka Y, Yamachi N, Matsumoto S, Kaneko S, Okabe S and Shibuya M 2008 Thermodynamic and transport properties of $\mathrm{CO}_{2}, \mathrm{CO}_{2}-\mathrm{O}_{2}$ and $\mathrm{CO}_{2}-\mathrm{H}_{2}$ mixtures at temperatures of $300-30,000 \mathrm{~K}$ and at Pressures of 0.1-10 MPa Electrical Engineering in Japan, 163-4 18-29.

[19] Murphy A B 2001 Thermal plasmas in gas mixtures J. Phys. D: Appl. Phys. 34 R151-R173.

[20] Murphy A B and Arundell C J 1994 Transport coefficients of argon, nitrogen, oxygen, argonnitrogen, and argon-oxyen plasma Plasma Chem. Plasma Process. 14 451-90.

[21] Tanaka Y 2004 Two-temperature chemically non-equilibrium modelling of high-power $\mathrm{Ar}-\mathrm{N}_{2}$ inductively coupled plasma at atmospheric pressure J. Phys. D: Appl. Phys. 371190205.

[22] Murphy A B 2010 The effects of metal vapour in arc welding J. Phys. D: Appl. Phys. 43434001.

[23] Yos J M 1963 Transport properties of nitrogen, hydrogen, oxygen and air to 30,000 K Techinical Memorandum RAD-TM-63-7 AVCO Corporation

[24] Abrahamson A A 1969 Born-Mayr-type interatomic potential for neutral ground-state atoms with $\mathrm{Z}=2$ to $\mathrm{Z}=105$ Phys. Rev. 178 76-79.

[25] Monchick L 1959 Collision integrals for the exponential repulsive potential Phys. Fluids 2 695-700.

[26] Rapp. D and Francis W E 1962 Charge exchange between gaseous ions and atoms J. Chem. Phys. 37 2613-2645.

[27] Charles H C and William R B, Experimental transition probabilites for spectral line of seventy elements, National Bureau of Standards Monograph 53, July, 1962.

[28] NIST Atomic Spectra Database, National Institute of Standards and Technology, http://www.nist.gov/.

[29] M.W. Chase, Jr. et al., NIST-JANAF Thermochemical Tables, Fourth edition, J. Phys. Chem. Ref. Data, Monograph 9, 1998.

[30] Patankar S V 1980 Numerical Heat Transfer and Fluid Flow Hemisphere Pub. Corp. New York 
Table 1. Thermodynamic properties of the solid material.

\begin{tabular}{ccccccccc}
\hline Solid & $\begin{array}{c}\rho_{\mathrm{s}} \\
{\left[\mathrm{kg} / \mathrm{m}^{3}\right]}\end{array}$ & $\begin{array}{c}C_{\mathrm{ps}} \\
{[\mathrm{J} / \mathrm{kg} / \mathrm{K}]}\end{array}$ & $\begin{array}{c}\lambda_{\mathrm{s}} \\
{[\mathrm{W} / \mathrm{m} \cdot \mathrm{K}]}\end{array}$ & $\begin{array}{c}T_{\text {melt }} \\
{[\mathrm{K}]}\end{array}$ & $\begin{array}{c}T_{\text {boil }}[\mathrm{K}] \\
{[\mathrm{MJ} / \mathrm{kg}]}\end{array}$ & $\begin{array}{c}L_{\mathrm{m}} \\
{[\mathrm{MJ} / \mathrm{kg}]}\end{array}$ & $\begin{array}{c}L_{\mathrm{v}} \\
{[\mathrm{eV}]}\end{array}$ \\
\hline $\mathrm{Cu}$ & 8930 & 385.62 & 381 & 1356 & 2855 & 0.206 & 4.815 & 4.65 \\
$\mathrm{Hf}$ & 13310 & 140 & 23 & 2506 & 4876 & 0.1347 & 3.211 & 3.53 \\
\hline
\end{tabular}



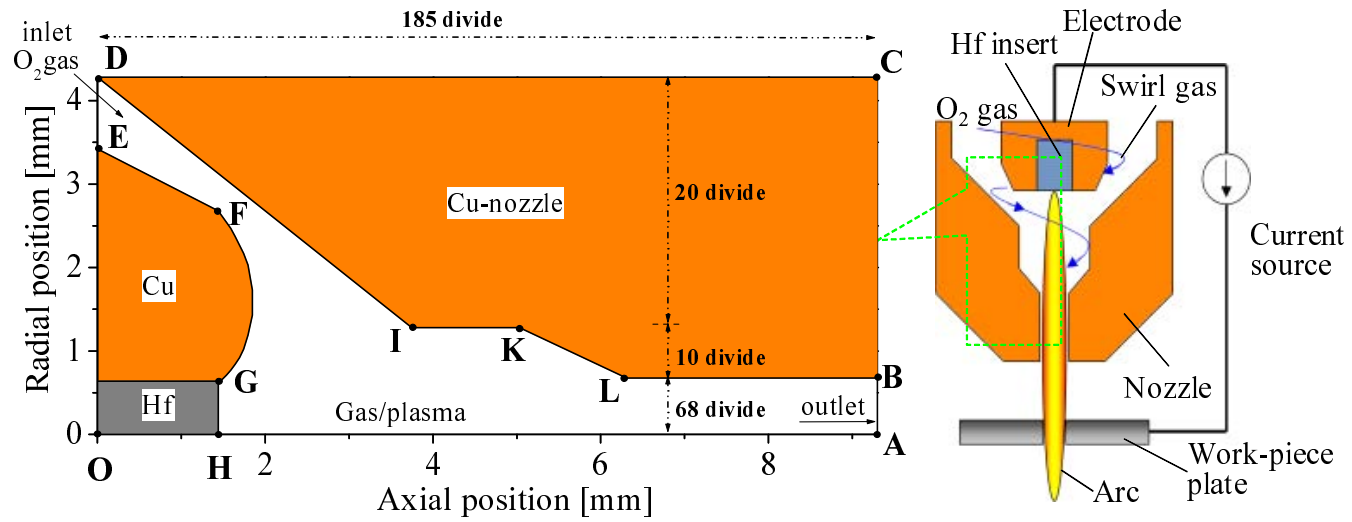

Figure 1. Schematic diagram of plasma cutting torch 

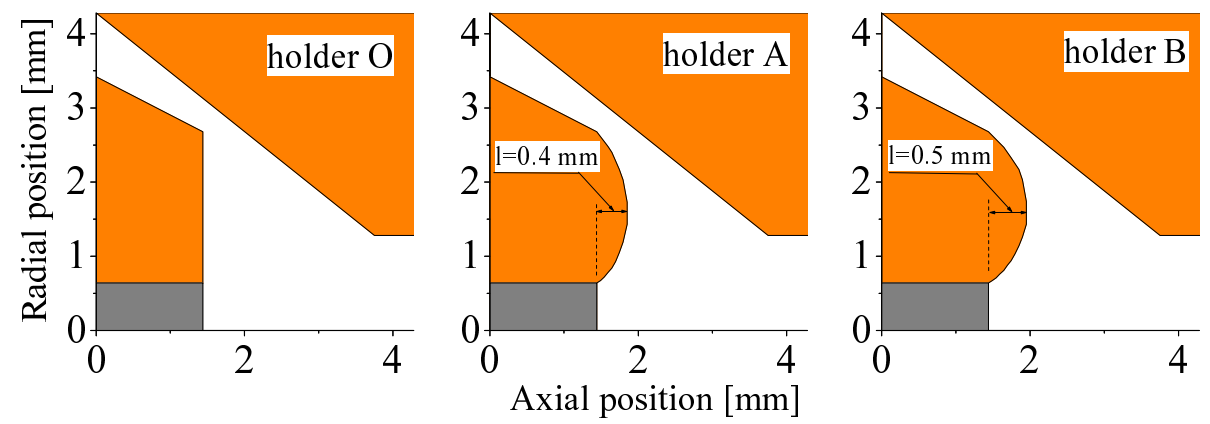

Figure 2. The shape of cathode holder. 


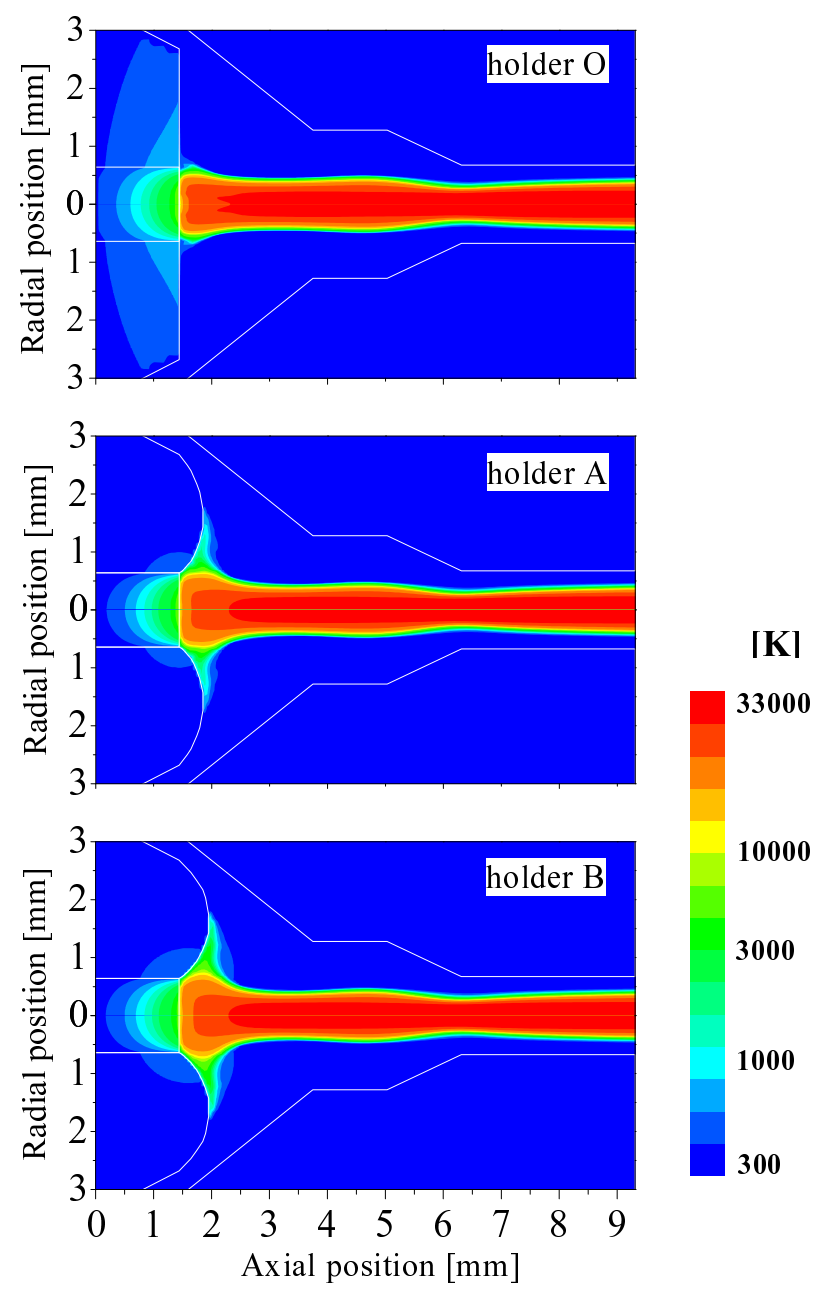

Figure 3. Temperature distribution of arc plasma with different cathode holder shapes. 


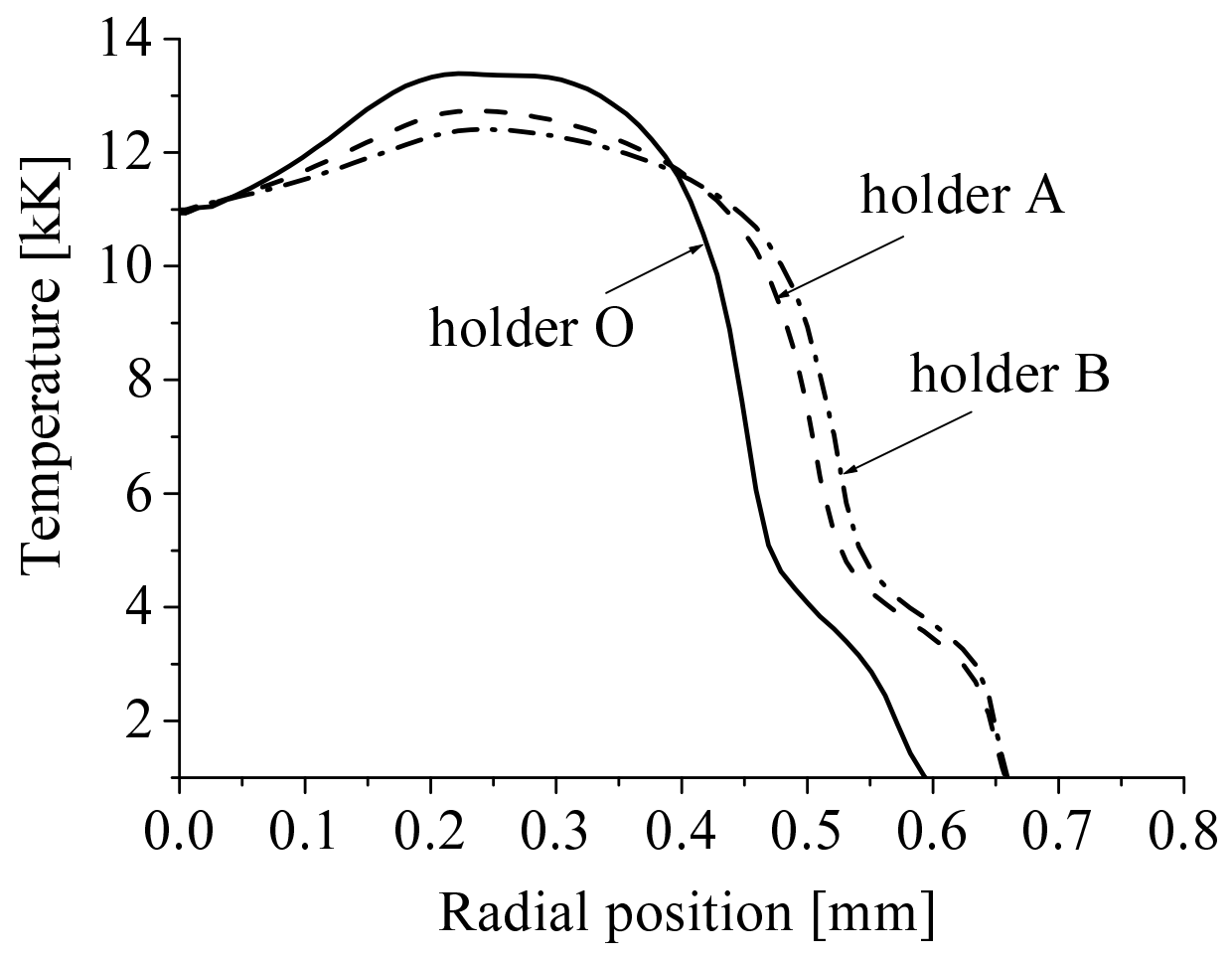

Figure 4. Radial distributions of the arc plasma temperature near the cathode surface for different cathode holder shapes. 


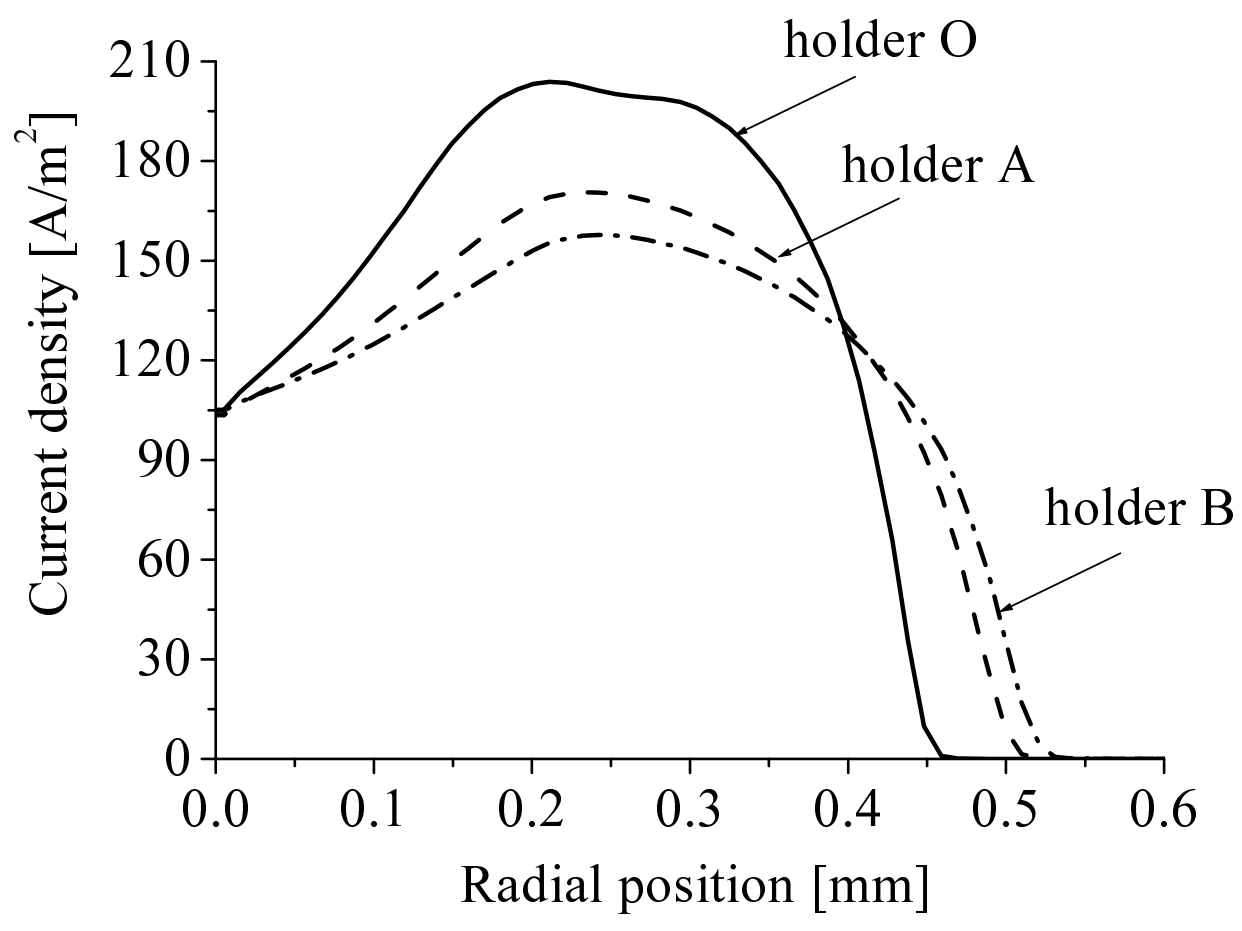

Figure 5. Radial distributions of current density near the cathode surface for different cathode holder shapes. 


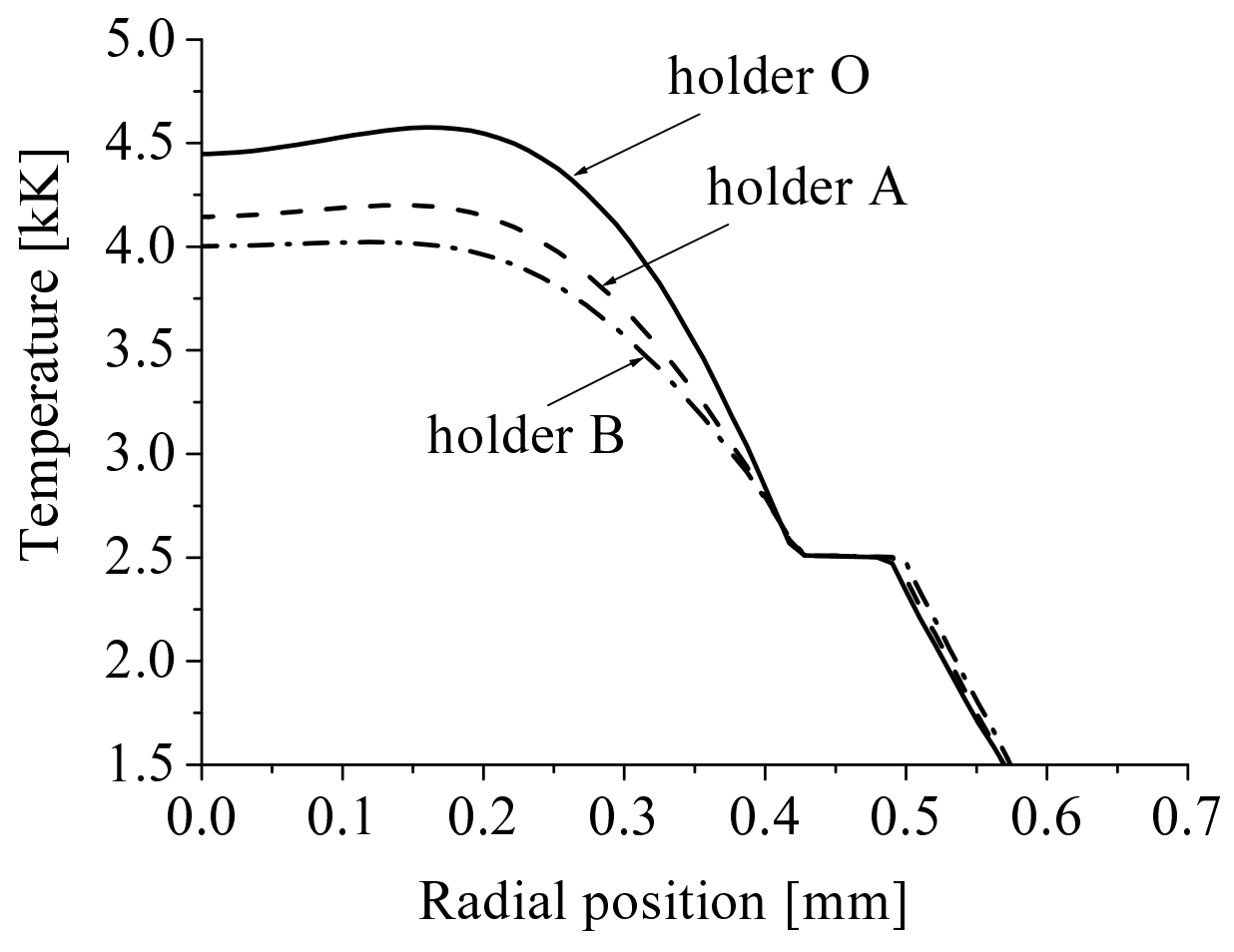

Figure 6. Radial distributions of the Hf cathode surface temperature for different cathode holder shapes. 

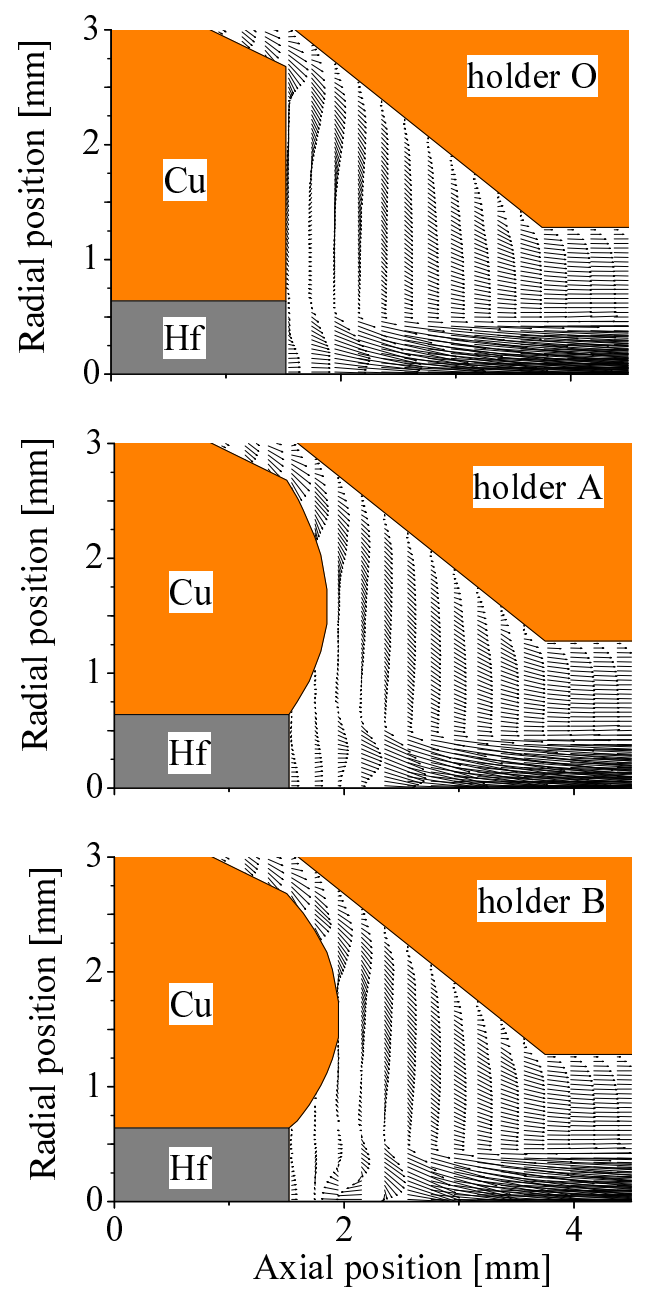

Figure 7. Gas flow fields with different cathode holder shapes. 


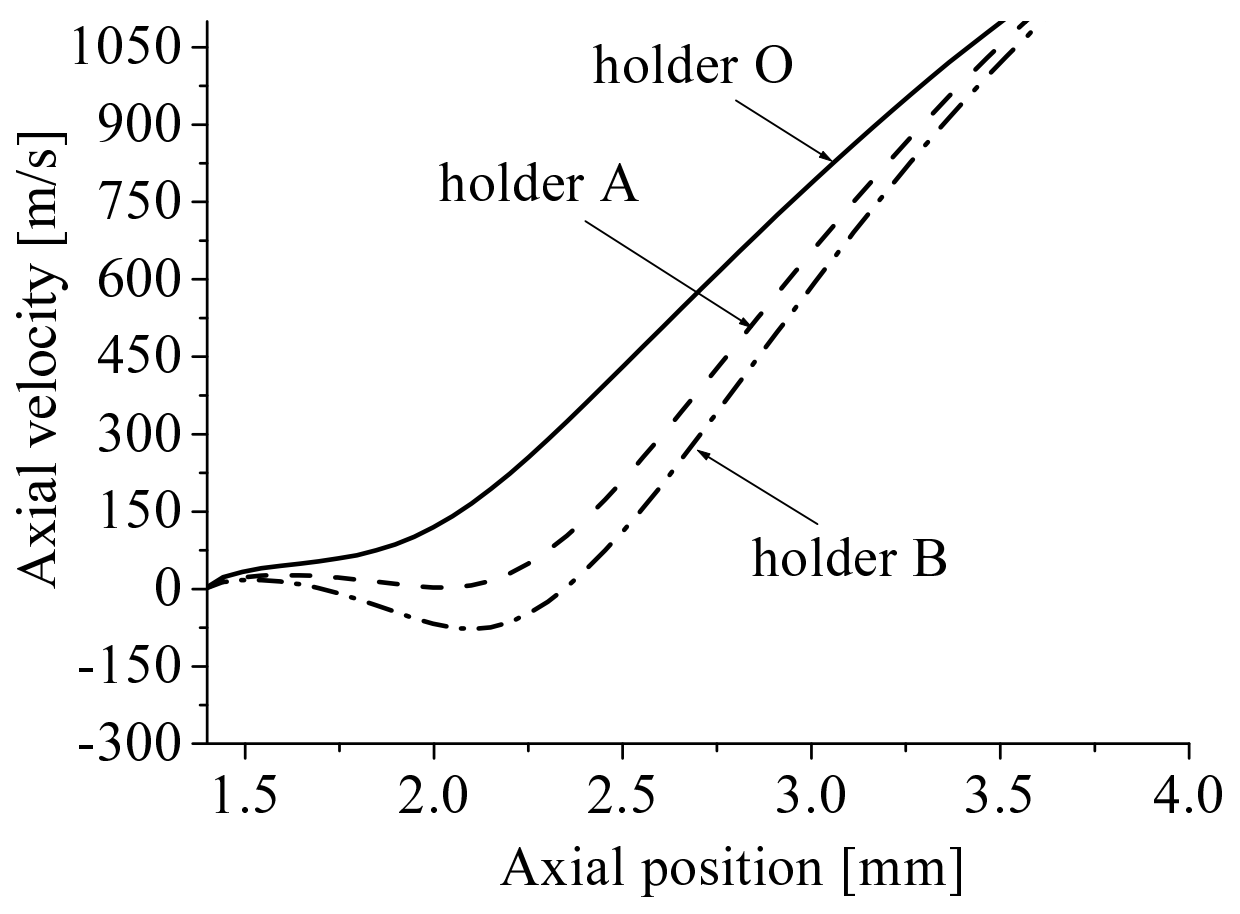

Figure 8. Axial distributions of velocity along the center axis for different cathode holder shapes. 

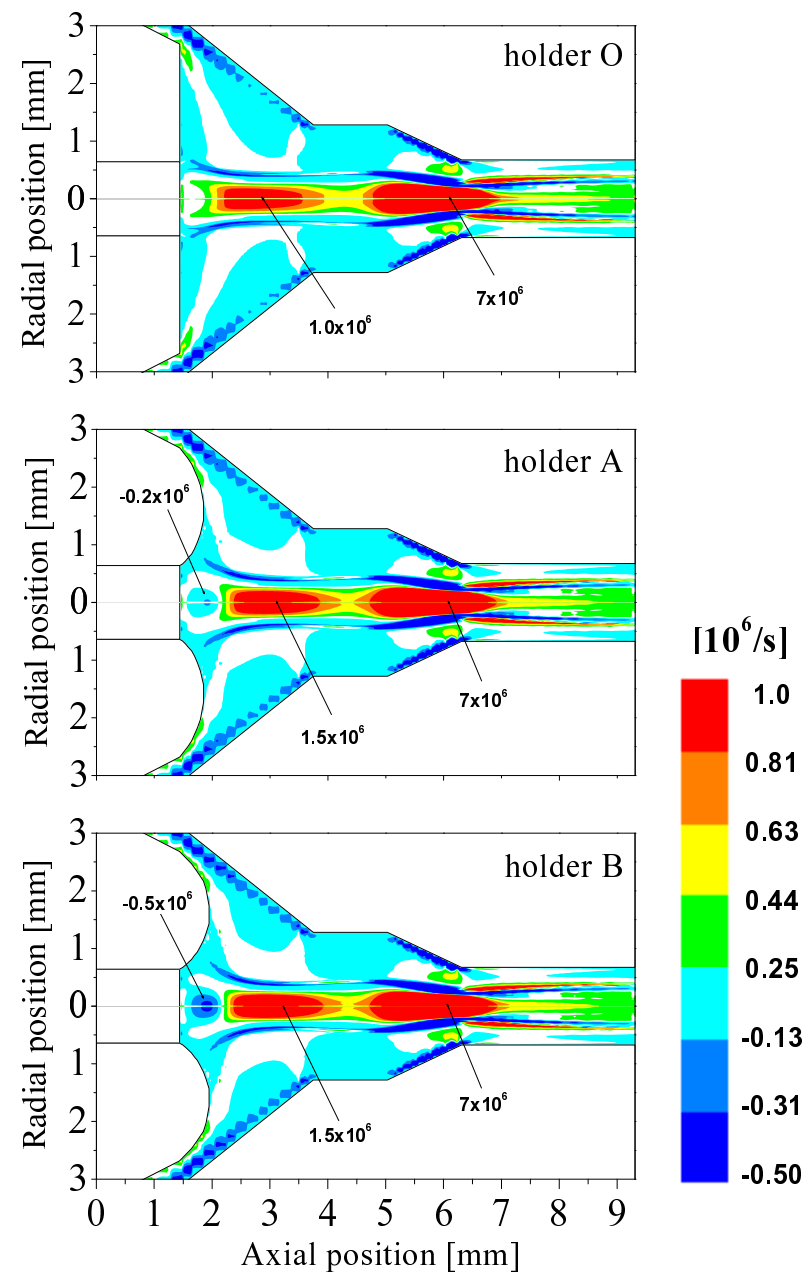

Figure 9. Distributions of vorticity with different cathode holder shapes. 
1E-5 3E-5 8E-5 2E-4 6E-4 2E-3 4E-3 1E-2 3E-2 9E-2 3E-1
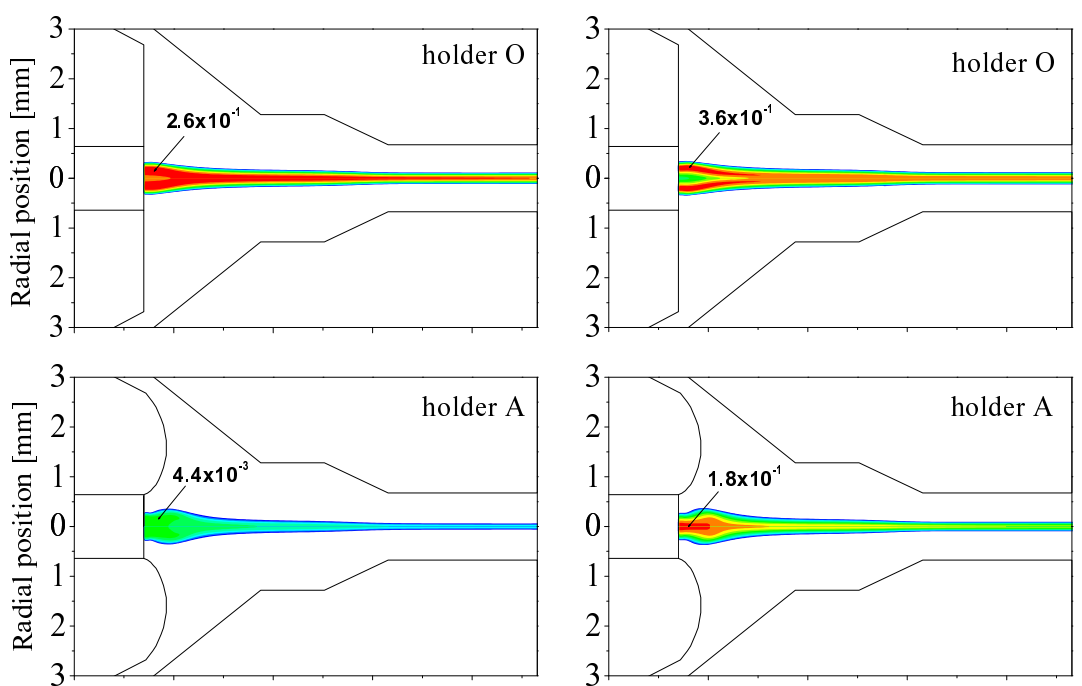

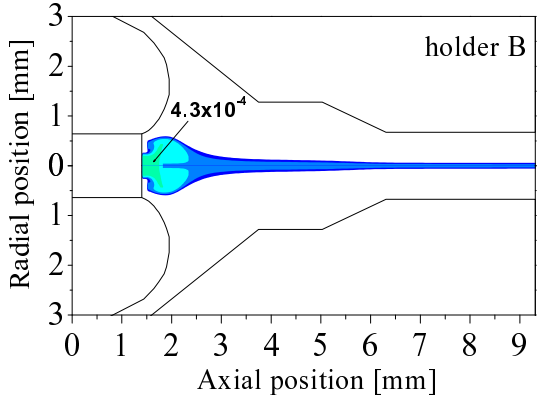

(i)

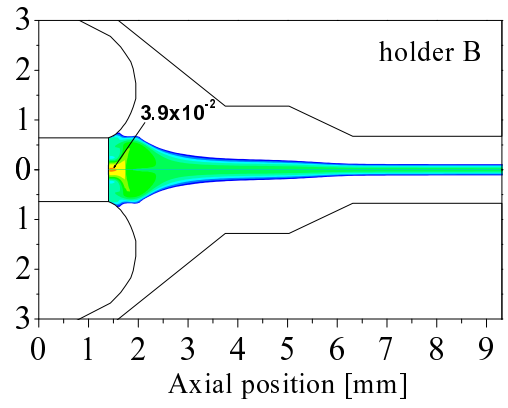

(ii)

Figure 10. Distributions of mass fraction of Hf vapour with different cathode holder shapes: (i) with redeposition; (ii) without redeposition. 


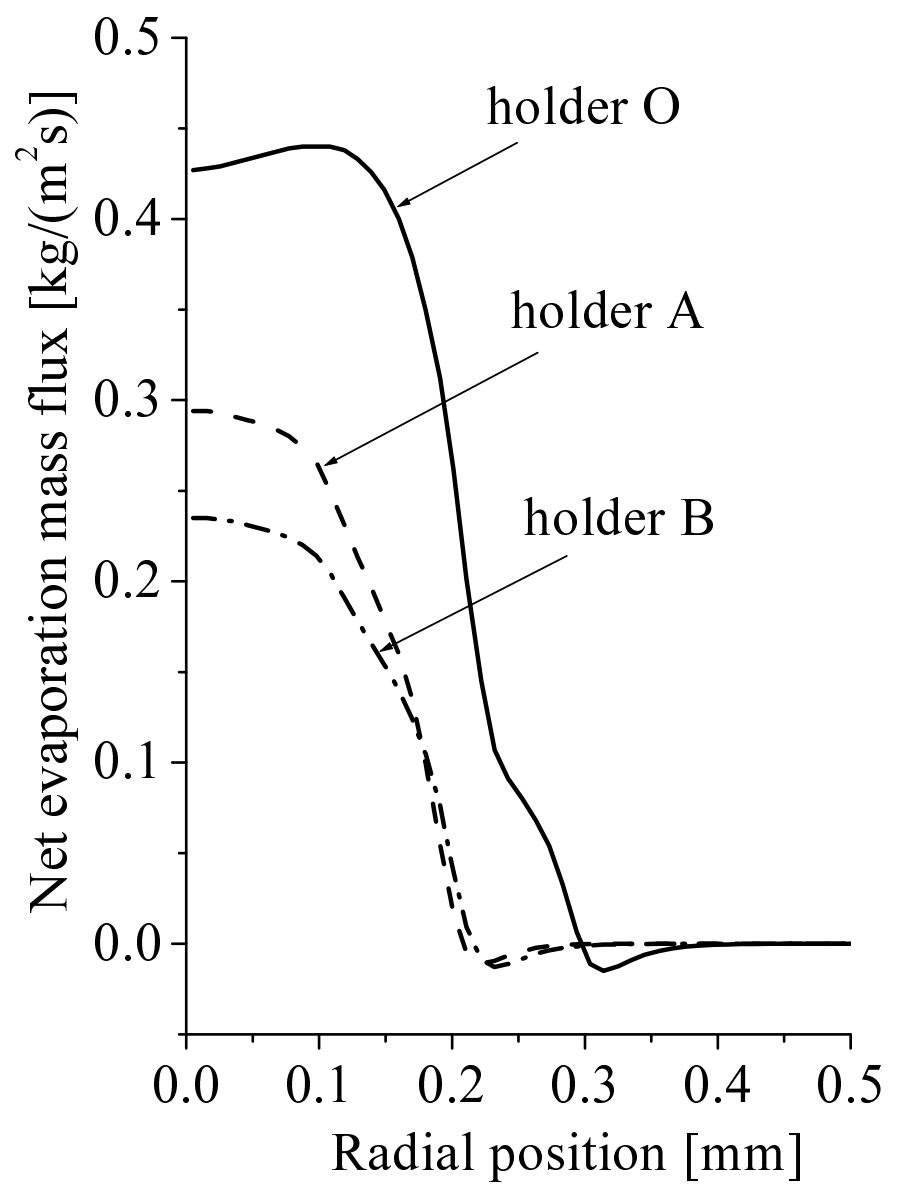

Figure 11. Radial distributions of net evaporated mass flux from Hf cathode for different cathode holder shapes. 


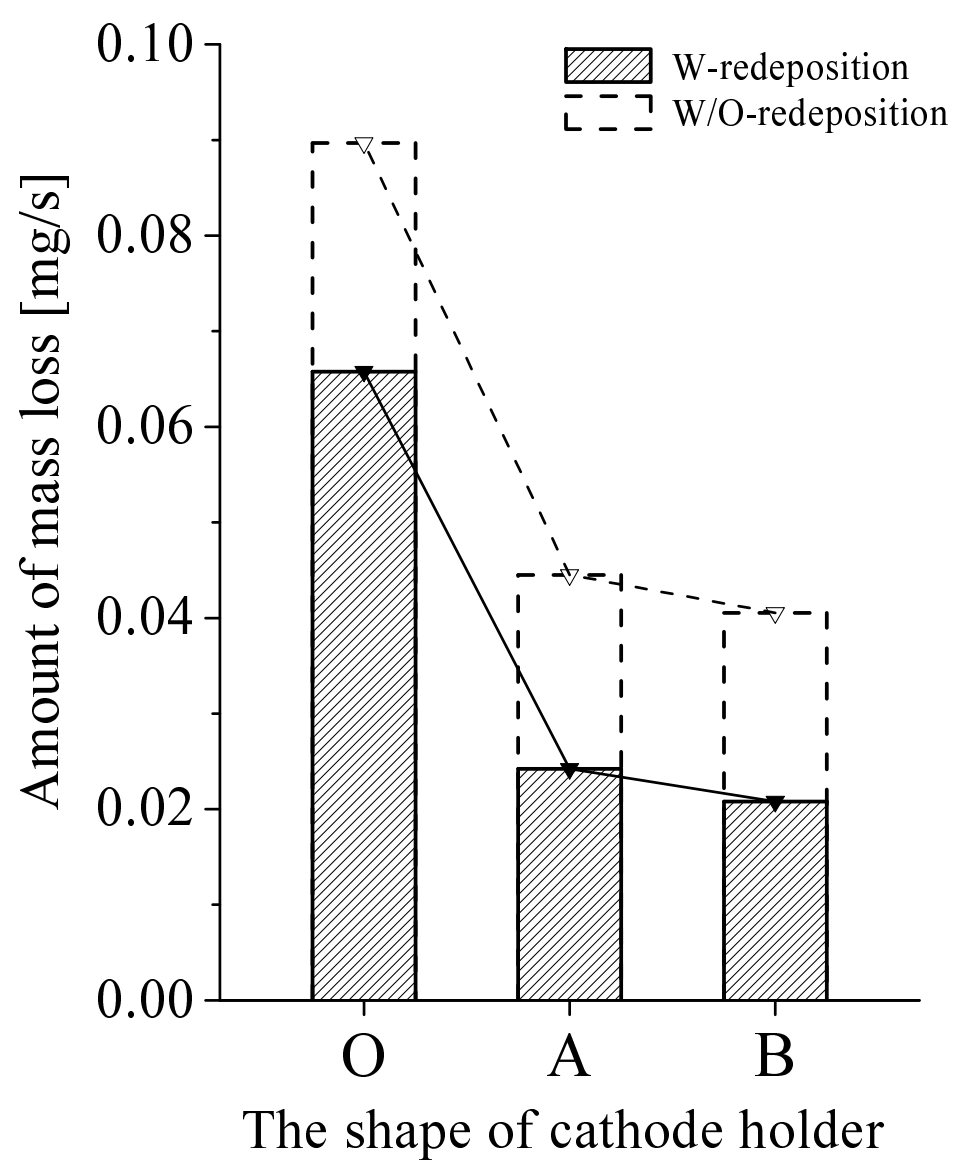

Figure 12. Total amounts of mass loss of Hf cathode evaporation for different cathode holder shapes. 